\section{This paper is published as part of a $P C C P$ themed issue on recent developments in X-ray absorption spectroscopy}

\section{Guest Editor: Jeroen Anton van Bokhoven}

Editorial

Recent developments in X-ray absorption spectroscopy J. A. van Bokhoven, Phys. Chem. Chem. Phys., 2010 DOI: $10.1039 / \mathrm{c0cp90010a}$

Perspectives

Parameter-free calculations of X-ray spectra with FEFF9 John J. Rehr, Joshua J. Kas, Fernando D. Vila, Micah P. Prange and Kevin Jorissen, Phys. Chem. Chem. Phys., 2010

DOI: $\underline{10.1039 / \mathrm{b} 926434 \mathrm{e}}$

The atomic AXAFS and $\Delta \mu$ XANES techniques as applied to heterogeneous catalysis and electrocatalysis D. E. Ramaker and D. C. Koningsberger, Phys. Chem. Chem. Phys., 2010

DOI: $\underline{10.1039 / \mathrm{b} 927120 \mathrm{c}}$

Advances in high brilliance energy dispersive X-ray absorption spectroscopy

Sakura Pascarelli and Olivier Mathon, Phys. Chem. Chem. Phys., 2010

DOI: $10.1039 / \mathrm{b} 926509 \mathrm{k}$

Communication

$\mu$-XANES mapping of buried interfaces: pushing microbeam techniques to the nanoscale

Paolo Ghigna, Sonia Pin, Giorgio Spinolo, Mark A. Newton, Michele Zema, Serena C. Tarantino, Giancarlo Capitani and Francesco Tatti, Phys. Chem. Chem. Phys., 2010 DOI: $10.1039 / \mathrm{c000195c}$

Papers

L-edge XANES analysis of photoexcited metal complexes in solution

Renske M. van der Veen, Joshua J. Kas, Christopher J. Milne, Van-Thai Pham, Amal El Nahhas, Frederico A. Lima, Dimali A. Vithanage, John J. Rehr, Rafael Abela and Majed Chergui, Phys. Chem. Chem. Phys., 2010

DOI: $10.1039 / \mathrm{b} 927033 \mathrm{~g}$

EXAFS as a tool to interrogate the size and shape of mono and bimetallic catalyst nanoparticles

Andrew M. Beale and Bert M. Weckhuysen, Phys. Chem.

Chem. Phys., 2010

DOI: $\underline{10.1039 / \mathrm{b} 925206 \mathrm{a}}$

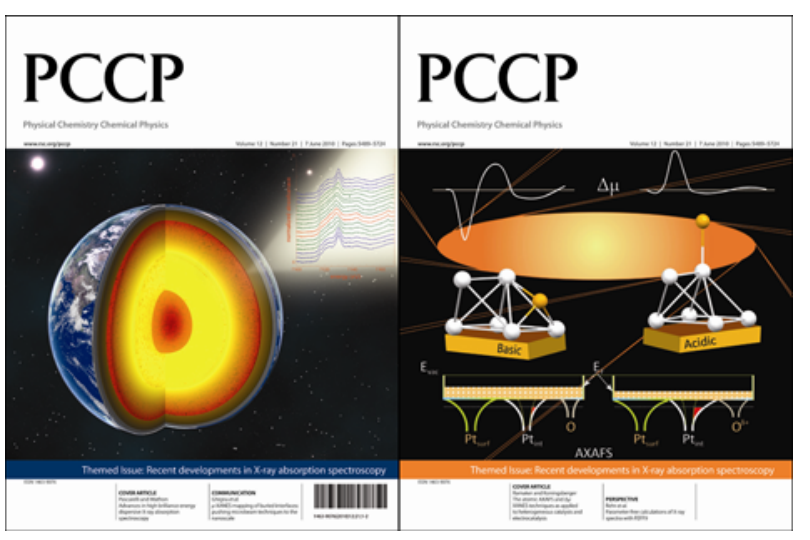

X-Ray absorption in homogeneous catalysis research: the iron-catalyzed Michael addition reaction by XAS, RIXS and multi-dimensional spectroscopy Matthias Bauer and Christoph Gastl, Phys. Chem. Chem. Phys., 2010

DOI: $10.1039 / \mathrm{b} 926385 \mathrm{c}$

Combined TPRx, in situ GISAXS and GIXAS studies of model semiconductor-supported platinum catalysts in the hydrogenation of ethene

Sonja A. Wyrzgol, Susanne Schäfer, Sungsik Lee,

Byeongdu Lee, Marcel Di Vece, Xuebing Li, Sönke Seifert, Randall E. Winans, Martin Stutzmann, Johannes A. Lercher and Stefan Vajda, Phys. Chem. Chem. Phys., 2010 DOI: $\underline{10.1039 / \mathrm{b} 926493 \mathrm{k}}$

Near sulfur L-edge X-ray absorption spectra of methanethiol in isolation and adsorbed on a Au(111) surface: a theoretical study using the four-component static exchange approximation

Sebastien Villaume, Ulf Ekström, Henrik Ottosson and Patrick Norman, Phys. Chem. Chem. Phys., 2010 DOI: $\underline{10.1039 / b 926109 e}$

Influence of additives in defining the active phase of the ethylene oxychlorination catalyst

N. B. Muddada, U. Olsbye, L. Caccialupi, F. Cavani, G. Leofanti, D. Gianolio, S. Bordiga and C. Lamberti, Phys. Chem. Chem. Phys., 2010

DOI: $10.1039 / \mathrm{b} 926502 \mathrm{n}$

First-principles calculations of X-ray absorption spectra at the K-edge of $3 d$ transition metals: an electronic structure analysis of the pre-edge Delphine Cabaret, Amélie Bordage, Amélie Juhin, Mounir Arfaoui and Emilie Gaudry, Phys. Chem. Chem. Phys., 2010

DOI: $10.1039 / \mathrm{b} 926499 \mathrm{j}$

First steps in combining modulation excitation spectroscopy with synchronous dispersive EXAFS/DRIFTS/mass spectrometry for in situ time resolved study of heterogeneous catalysts Davide Ferri, M. Santosh Kumar, Ronny Wirz, Arnim Eyssler, Oxana Korsak, Paul Hug, Anke Weidenkaff and Mark A. Newton, Phys. Chem. Chem. Phys., 2010 DOI: $10.1039 / \mathrm{b} 926886 \mathrm{c}$

Novel opportunities for time-resolved absorption spectroscopy at the X-ray free electron laser B. D. Patterson and R. Abela, Phys. Chem. Chem. Phys., 2010

DOI: $10.1039 / \mathrm{c003406a}$ 
Spatially resolved 3D micro-XANES by a confocal detection scheme

Geert Silversmit, Bart Vekemans, Sergey Nikitenko, Sylvia

Schmitz, Tom Schoonjans, Frank E. Brenker and Laszlo

Vincze, Phys. Chem. Chem. Phys., 2010

DOI: $\underline{10.1039 / \mathrm{c004103n}}$

Wavelet transform EXAFS analysis of mono- and dimolybdate model compounds and a Mo/HZSM-5

dehydroaromatization catalyst

Robert O. Savinelli and Susannah L. Scott, Phys. Chem.

Chem. Phys., 2010

DOI: $\underline{10.1039 / \mathrm{b} 926474 d}$

Electronic structure of alumina-supported monometallic Pt and bimetallic PtSn catalysts under hydrogen and carbon monoxide environment

Jagdeep Singh, Ryan C. Nelson, Brian C. Vicente,

Susannah L. Scott and Jeroen A. van Bokhoven, Phys.

Chem. Chem. Phys., 2010

DOI: $10.1039 / \mathrm{c} 000403 \mathrm{k}$

\section{Determination of $\mathrm{CO}, \mathrm{H}_{2} \mathrm{O}$ and $\mathrm{H}_{2}$ coverage by XANES} and EXAFS on $\mathrm{Pt}$ and Au during water gas shift reaction Neng Guo, Bradley R. Fingland, W. Damion Williams, Vincent F. Kispersky, Jelena Jelic, W. Nicholas Delgass, Fabio H. Ribeiro, Randall J. Meyer and Jeffrey T. Miller, Phys. Chem. Chem. Phys., 2010

DOI: $\underline{10.1039 / \mathrm{c000240m}}$

Complementarity between high-energy photoelectron and L-edge spectroscopy for probing the electronic structure of $5 \mathrm{~d}$ transition metal catalysts

Toyli Anniyev, Hirohito Ogasawara, Mathias P. Ljungberg, Kjartan T. Wikfeldt, Janay B. MacNaughton, Lars-Åke Näslund, Uwe Bergmann, Shirlaine Koh, Peter Strasser, Lars G.M. Pettersson and Anders Nilsson, Phys. Chem. Chem. Phys., 2010

DOI: $10.1039 / \mathrm{b} 926414 \mathrm{k}$

In situ time-resolved DXAFS for the determination of kinetics of structural changes of H-ZSM-5-supported active Re-cluster catalyst in the direct phenol synthesis from benzene and $\mathrm{O}_{2}$

Mizuki Tada, Yohei Uemura, Rajaram Bal, Yasuhiro Inada, Masaharu Nomura and Yasuhiro Iwasawa, Phys. Chem.

Chem. Phys., 2010

DOI: $10.1039 / \mathrm{c000843p}$

Sulfur poisoning mechanism of steam reforming catalysts: an X-ray absorption near edge structure (XANES) spectroscopic study

Yongsheng Chen, Chao Xie, Yan Li, Chunshan Song and

Trudy B. Bolin, Phys. Chem. Chem. Phys., 2010

DOI: $10.1039 / \mathrm{b} 925910 \mathrm{~b}$

Peroxide-like intermediate observed at hydrogen rich condition on $\mathrm{Pt}(111)$ after interaction with oxygen

Janay B. MacNaughton, Lars-Åke Näslund, Toyli Anniyev, Hirohito Ogasawara and Anders Nilsson, Phys. Chem.

Chem. Phys., 2010

DOI: 10.1039/b926409b 


\title{
Spatially resolved 3D micro-XANES by a confocal detection scheme
}

\author{
Geert Silversmit, ${ }^{* a}$ Bart Vekemans, ${ }^{a}$ Sergey Nikitenko, ${ }^{b}$ Sylvia Schmitz, ${ }^{c}$ \\ Tom Schoonjans, ${ }^{a}$ Frank E. Brenker ${ }^{c}$ and Laszlo Vincze ${ }^{a}$
}

Received 9th March 2010, Accepted 6th April 2010

First published as an Advance Article on the web 29th April 2010

DOI: $10.1039 / \mathbf{c 0 0 4 1 0 3 n}$

\begin{abstract}
A confocal setup based on polycapillary half-lenses was used to demonstrate three-dimensional (3D) spatially resolved $\mu$-XANES in fluorescence detection mode at the DUBBLE XAS station of the ESRF (BM26A). The incoming beam was focused using a polycapillary half-lens and a second glass polycapillary was placed in front of the energy dispersive detector to establish the confocal detection. The full-width-half-maxima along the main axes of the resulting ellipsoidal detection volume were $18.5 \times 12.0 \times 10.0 \mu \mathrm{m}^{3}$ at the $\mathrm{Cu}$ K-edge. The confocal $\mu$-XANES mode is applied in the $3 \mathrm{D}$ resolved study of mineral inclusions in rare natural diamonds at the Fe K edge.
\end{abstract}

\section{Introduction}

Standard XAS experiments are typically performed on homogeneous or homogenised samples with millimetre sized $\mathrm{X}$-ray beams in transmission or fluorescence detection mode. However, in various research disciplines, such as environmental sciences, Earth and planetary sciences, cultural heritage studies etc., microscopically inhomogeneous samples are very often of interest. Spatially resolved spectroscopic information can be obtained on such heterogeneous samples by using $\mathrm{X}$-ray beams with micrometre dimensions. Sub-millimetre X-ray beams are routinely achieved by applying different focusing lenses such as compound refractive lenses, Fresnel lenses or mirror based techniques such as saggital focusing and Kirkpatrick-Baez (KB) systems. Recently, we evaluated the analytical characteristics with respect to $\mu$-EXAFS and $\mu$-XANES at a standard 3rd generation bending magnet XAFS station without any prefocusing using a polycapillary half-lens. ${ }^{1}$ Polycapillaries consist of a bundle of several hundred thousands of narrow bent glass fibres oriented towards the same focal point. X-rays entering the glass fibres are guided towards that focal point by repeated total reflections which results in an achromatic optical element with respect to the focal distance. With this polycapillary based $\mu$-setup transmission efficiencies of $25-45 \%$ with flux density gain factors of about 2000 and beam sizes of 10-20 $\mu \mathrm{m}$ were obtained in the $7-14 \mathrm{keV}$ energy range. ${ }^{1}$ The achieved beam sizes are smaller compared to monochromator saggital focusing (typically $150 \times 200 \mu \mathrm{m}^{2}(\mathrm{~V} \times \mathrm{H})$ ) and comparable to or somewhat larger than KB systems (typically $10 \times 10 \mu \mathrm{m}^{2}$ ).

By scanning a heterogeneous sample through a $\mu$-beam along a $2 \mathrm{D}$ raster, a two-dimensional, spatially resolved analysis is possible. In this case the information originates

${ }^{a} X$-Ray Microspectroscopy and Imaging Research Group (XMI), Department of Analytical Chemistry, Ghent University, Krijgslaan 281 S12, B-9000 Gent, Belgium.E-mail: Geert.Silversmit@UGent.be

${ }^{b}$ Netherlands Organisation for Scientific Research (NWO), DUBBLE at ESRF, BP 220, 38043 Grenoble CEDEX 9, France

${ }^{c}$ Geoscience Institute-Mineralogy, Goethe University, Altenhoeferallee 1, 60438 Frankfurt am Main, Germany from the intersection path of the beam through the sample (limited by the signal absorption length) and no depth information of the sample is obtained (see Fig. 1a). Glass polycapillary half-lenses can be used to realize a confocal detection scheme in the fluorescence detection mode which allows to obtain spatially resolved $\mu$-XANES in $3 \mathrm{D}$. In this confocal detection mode, a polycapillary half-lens is placed in front of the energy dispersive detector in order to strongly reduce the acceptance path 'seen' by the detector. The detected volume is now determined by the coinciding foci of the incoming $\mu$-beam and the detector polycapillary, see Fig. $1 b$.

The focusing polycapillary has a depth of focus of about $200 \mu \mathrm{m}$ and delivers a $\mu$-beam with a symmetric binormal intensity distribution around the beam direction in the focal plane. ${ }^{1}$ For the polycapillary at the detector side a binormal acceptance around the detector axis can therefore be assumed. ${ }^{2}$ Combining these binormal distributions results in an ellipsoidal shaped detection volume. For a perfectly aligned confocal setup the vertical full-width-at-half-maximum (FWHM), $D_{\mathrm{V}}$, of the ellipsoidal detection volume is given by $\left(1 / D_{\mathrm{V}}\right)^{2}=\left(1 / D_{\mathrm{A}}\right)^{2}+\left(1 / D_{\mathrm{B}}\right)^{2},{ }^{2}$ with $D_{\mathrm{B}}$ the FWHM of the focused beam and $D_{\mathrm{A}}$ the (energy dependent) acceptance FWHM, see Fig. 1c.

We present the first confocal $\mu$-XANES data for a 3D resolved $\mathrm{Fe} \mathrm{K} \mu$-XANES study on mineral inclusions within rare natural diamonds. The confocal detection is based on using a pair of polycapillary optics for focusing the incoming beam and collimating the detector. The confocal setup is characterised on the basis of its analytical elemental detection limits and the FWHM values of the ellipsoidal detection volume.

\section{Experimental}

The confocal XANES experiments were performed at the DUBBLE beamline (BM26A) ${ }^{3}$ of the ESRF (Grenoble, France) and at beamline $\mathrm{L}$ at HASYLAB (Hamburg, Germany). The characterisation of the confocal $\mu$-setup is focused on the data obtained at the DUBBLE beamline. 


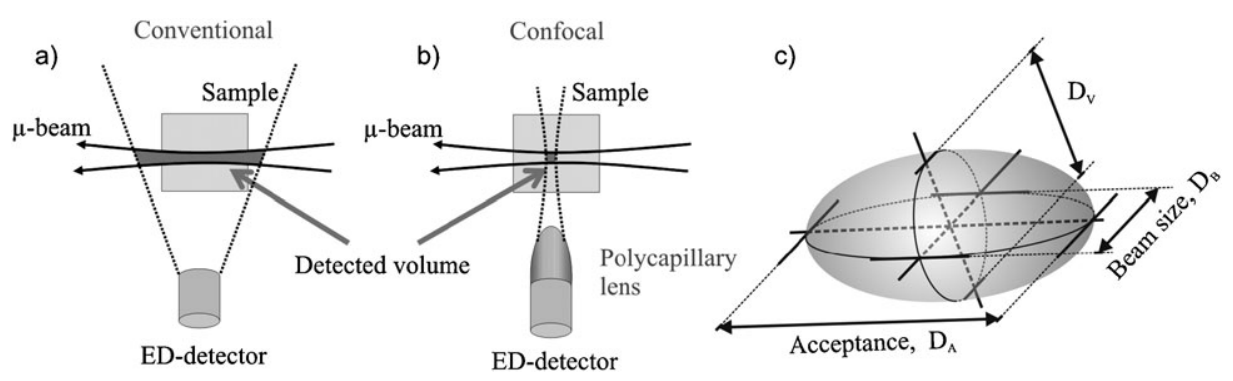

Fig. 1 (a) Conventional fluorescence detection, (b) confocal fluorescence detection and (c) ellipsoidal detection volume for the confocal detection mode.

\subsection{DUBBLE beamline, ESRF}

The measurements at BM26A were performed during a uniform filling mode of the $6 \mathrm{GeV}$ storage ring, typically yielding ring currents of 200 down to $160 \mathrm{~mA}$ during a single synchrotron run. The DUBBLE XAS beamline receives a total of $2 \mathrm{mrad}$ of the bending magnet radiation fan (magnetic field strength $B=0.4$ Tesla, critical energy $E_{\mathrm{c}}=9.6 \mathrm{keV}$ ). The horizontal and vertical divergences of the source are $103 \mu \mathrm{rad}$ and $1.1 \mu \mathrm{rad}$ respectively. The beamline is equipped with a non fixed-exit $\mathrm{Si}(111)$ double crystal sagittal focusing monochromator, giving an energy resolution of $\Delta E / E=$ $2 \times 10^{-4}$. The higher harmonics of the primary energy were suppressed by a Si reflecting strip on a vertical focusing mirror after the monochromator. This mirror has a surface roughness of 1.5 Angstrom rms and $1.5 \mu \mathrm{rad}$ slope error and is placed under a grazing incidence angle of $2.8 \mathrm{mrad}$. The saggital focusing capability of the monochromator was not used during the experiments and no bending was applied to the vertical focusing mirror, which served as a flat mirror.

The incoming X-ray beam with dimensions of about $0.5 \times$ $2 \mathrm{~mm}^{2}(\mathrm{~V} \times \mathrm{H})$ was then focused using a large acceptance polycapillary half-lens with an entrance diameter of $5 \mathrm{~mm}$, an optic length of $50 \mathrm{~mm}$, an exit diameter of $1.6 \mathrm{~mm}$ and a focal distance of $3.6 \mathrm{~mm}$ from the exit tip of the optic, manufactured by XOS (X-Ray Optical Systems Inc., USA). This lens was specifically designed for the beam characteristics of DUBBLE (i.e. with respect to source size/divergence, source-sample distance, bending magnet energy range). The beam focusing polycapillary is placed in between the first ionisation chamber and the sample. A second glass polycapillary also from XOS (focal distance $2.2 \mathrm{~mm}$, output diameter $5.5 \mathrm{~mm}$, optical length $35 \mathrm{~mm}$ ) was mounted in front of the energy dispersive detector to achieve the confocal detection geometry.

The intensity of the incoming and transmitted X-ray beams were measured using two ionisation chambers (Oxford Instrument, UK). A Vortex-EM silicon drift detector (Si SDD) was used for the fluorescence radiation detection in the conventional and confocal mode. This energy dispersive detector has an energy resolution of typically $<136 \mathrm{eV}$ FWHM at Mn K $\alpha$.

A top view of the experimental setup used at BM26A is given in Fig. 2, with the synchrotron radiation emerging from the right. After passing the first ionisation chamber, the $\mathrm{X}$-rays were focused by the first polycapillary half-lens. A motor assembly consisting of two translation motors (up-down and left-right in the plane of the synchrotron), a rotation and a cradle motor was used to align the focusing polycapillary with the incoming X-ray beam. The sample was placed on a goniometer head fixed to a $X Y Z$ and rotation stage. The fluorescence detector with the polycapillary collimator was placed on a $X Y Z$ motor assembly to allow the confocal alignment.

The use of the optical microscope allows easy positioning of the sample. The focal plane of the optical microscope is set so that it coincides with the position of the $\mu$-beam, see Fig. 2c. A sample can then be easily aligned with respect to the $\mu$-beam by optimising its position until a sharp optical image is obtained.

The position of the focal plane and the vertical and horizontal beam sizes were determined by scanning a $\mathrm{Au}$ wire (diameter: $180 \mu \mathrm{m}$ ) through the X-ray beam. Knife-edge scans were obtained by recording the transmitted X-ray intensity, $I_{\mathrm{t}}$, with the second ionisation chamber as a function of the scanning position. The FWHM of the Gaussian fit to the first derivative of the knife-edge scans was taken as beam sizes.

For the confocal detection mode, metal wires of $10 \mu \mathrm{m}$ thickness were used to determine the axes of the detection ellipsoid, e.g. at the Fe K-edge a stainless steel wire was used.

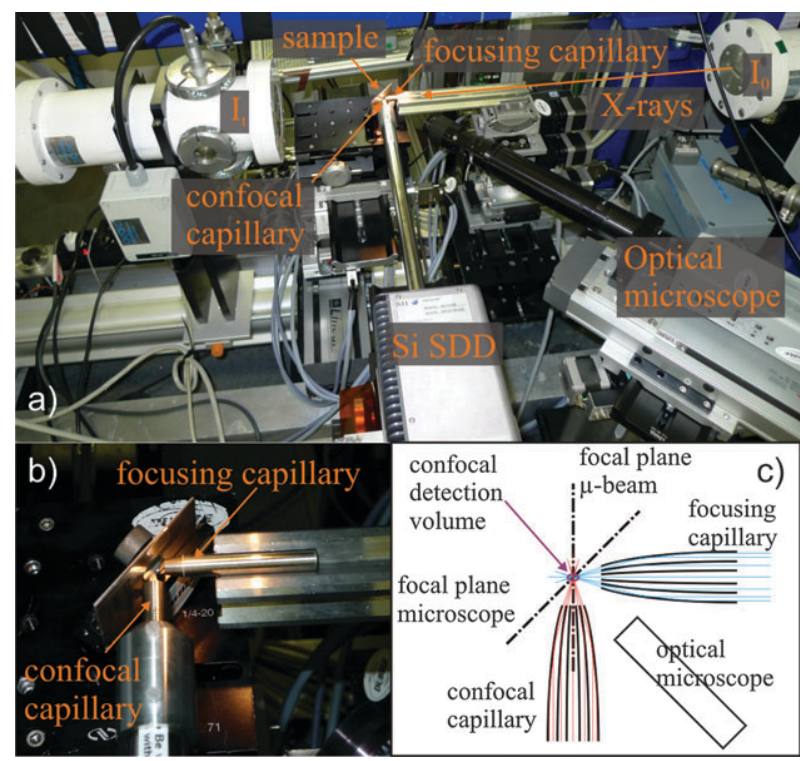

Fig. 2 (a) Top view of the confocal setup used at BM26A, (b) zoom around the sample showing the focusing and confocal polycapillary configuration, (c) schematic representation of the confocal and optical microscope configuration. 
These wire scans were obtained by moving the wire along the main axes of the ellipsoid and recording the fluorescence intensity, $I_{\mathrm{f}}$, as function of scanning position. Deconvolution of the measured profiles with the wire thickness gives the intensity distribution along the main axes. The FWHM of these deconvoluted intensity profiles are then taken as the FWHM of the main axis of the ellipsoidal confocal detection volume.

The detection limits in XRF-mode were determined using the NIST SRM 1577b (bovine liver) standard for a counting time of $300 \mathrm{~s}$ and excitation energies above the K-absorption edge energies of $\mathrm{Fe}, \mathrm{Cu}$ and $\mathrm{Zn}$ for both conventional and confocal microbeam setup. An amount of $18.7 \mathrm{mg}$ of NIST SRM 1577 b powder was pressed in a pellet with a diameter of $13 \mathrm{~mm}$. The absolute detection limits were calculated by taking into account the Gaussian intensity distribution of the incoming beam and of the ellipsoidal detection volume. The reported detection limits for the conventional fluorescence detection are corrected for self-absorption effects, using the fundamental constants tabulated in xraylib. ${ }^{4}$ The matrix composition for the SRM 1577b was estimated from the NIST certificate, adding the Hydrogen concentration from the NIST SRM1577c certificate, the follow-up of the SRM 1577b, balanced with oxygen and carbon in a 50/50 ratio. Balancing with only carbon or oxygen results in relative differences on the detection limit values of $25-50 \%$ for the lowest $\mathrm{Z}$ element (here $\mathrm{P}$ ), but these decrease rapidly for heavier elements and become less than $10 \%$ for the transition metals $\mathrm{Mn}-\mathrm{Cu}$.

\subsection{Beamline L, HASYLAB}

Beamline $\mathrm{L}$ is a bending magnet station (1.2 Tesla, critical energy: $16.04 \mathrm{keV}$ ) of the $4.44 \mathrm{GeV}$ positron storage ring DORIS III at HASYLAB (Hamburg, Germany). This beamline is dedicated to scanning $\mu-\mathrm{XRF}$, scanning confocal $\mu$-XRF and $\mu$-XANES and equipped with a Si(111) double crystal monochromator (energy resolution $\Delta E / E \sim 10^{-4}$ ) for XAS measurements. The double crystal was detuned to $70 \%$ for higher harmonics rejection. The incoming monochromatised $\mathrm{X}$-ray beam intensity is monitored with a $10 \mathrm{~cm}$ long ionisation chamber and focused with a large acceptance glass polycapillary manufactured by X-Ray Optical Systems (XOS Inc., focal distance of $4.8 \mathrm{~mm}$ ). The fluorescence radiation is detected with a Vortex-EX silicon drift detector (SII, $50 \mathrm{~mm}^{2}, 350 \mu \mathrm{m}$ crystal thickness, measured energy resolution $183 \mathrm{eV}$ at $\mathrm{Fe} \mathrm{K} \alpha$ ). The confocal detection was achieved by positioning a second polycapillary half-lens (XOS inc., focal distance $2.1 \mathrm{~mm}$ ) in front of the energy dispersive detector. A photograph of the confocal sample setup used can be found in. ${ }^{5}$

\section{Confocal $\mu$-setup characterisation}

\subsection{Beam size as a function of incident X-ray energy}

The vertical beam size as a function of the distance with respect to the exit window of the polycapillary lens was measured at an X-ray energy of $8.93 \mathrm{keV}$ to locate the focal plane of the focusing polycapillary, see Fig. 3. The vertical beam size at the $\mathrm{Fe}, \mathrm{Cu}$ and $\mathrm{Zn} \mathrm{K}$ absorption edges were 20.5, 18.3 and $16.4 \mu \mathrm{m}$ FWHM respectively.

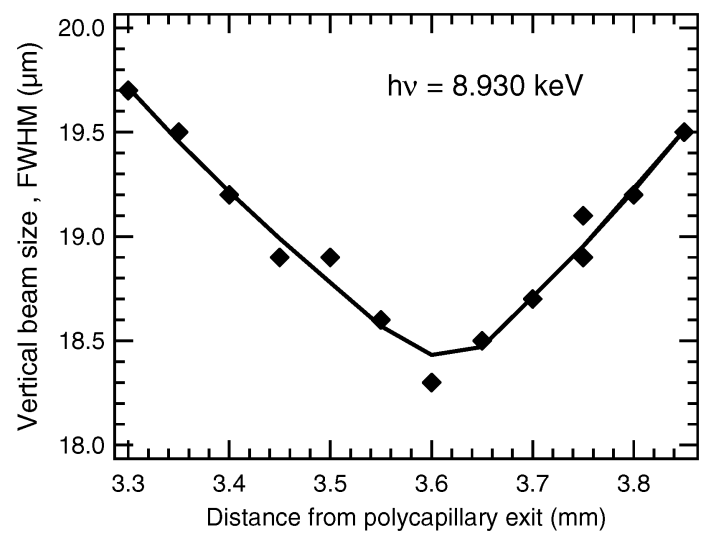

Fig. 3 Measured vertical FWHM $(\mu \mathrm{m})$ of the polycapillary focused $\mu$-beam as function of the distance from the polycapillary exit window at an incident X-ray energy of $8.930 \mathrm{keV}$.

\subsection{Confocal detection volume}

The FWHM of the ellipsoidal detection volume were determined at the $\mathrm{Fe} \mathrm{K}$ and at the $\mathrm{Cu} \mathrm{K}$ absorption edges. The measured acceptances for $\mathrm{Fe} \mathrm{K} \alpha$ and $\mathrm{Cu} \mathrm{K} \alpha$ radiation determined with wire scans were 18.5 and $12.0 \mu \mathrm{m}$ respectively. Together with the beam sizes given in section 3.1, this results in dimensions for the ellipsoidal detection volume at the $\mathrm{Fe}$ K-edge of $20.5 \times 18.5 \times 14.0 \mu^{3}\left(D_{\mathrm{B}} \times D_{\mathrm{A}} \times D_{\mathrm{V}}\right)$ and $18.5 \times 12.0 \times 10.0 \mu \mathrm{m}^{3}\left(D_{\mathrm{B}} \times D_{\mathrm{A}} \times D_{\mathrm{V}}\right)$ at the $\mathrm{Cu}$ K-edge.

\subsection{Elemental detection limits}

The elemental minimum detection limits for the conventional $\mu$-XRF setup for an excitation energy of $9.027 \mathrm{keV}$ and a measuring time of $300 \mathrm{~s}$ are given in Fig. 4. The detection limits with and without correcting for self-absorption effects are compared. For the $3 \mathrm{~d}$ transition metals probed $(\mathrm{Mn}-\mathrm{Cu})$ relative detection of about $0.1 \mathrm{ppm}$ are obtained in the fluorescence detection mode, with absolute detection limits of less than $10 \mathrm{fg}$. These detection limits are comparable with a typical dedicated bending magnet $\mu$-XRF instrument like HASYLAB-L, (see ref. 6 using a multilayer monochromator and an excitation energy of $20 \mathrm{keV}$ ).

The corresponding elemental detection limits for the confocal detection mode at the same excitation energy of $9.027 \mathrm{keV}$ are given in Fig. 5 and are similar or slightly less good compared to the conventional $\mu$-XRF detection limits given in Fig. 4.

\subsection{Comparative $\mu$-XANES spectra}

The $\mu$-XANES spectrum at the Zn K-edge of the NIST SRM1577b standard recorded in confocal detection mode is compared in Fig. 6 with the $\mu$-XANES spectrum obtained on the same sample in conventional fluorescence detection and with the conventional broad beam fluorescence spectrum. Apart from the noise level, the three spectra in Fig. 6 are comparable, the confocal detection mode does not introduce any artefacts in the $\mu$-XANES spectrum.

The conventional fluorescence spectrum in Fig. 6 has been recorded with a X-ray beam of $2.0 \times 0.5 \mathrm{~mm}^{2}$, the intersection volume of the X-ray beam with the pressed SRM1577b pellet 

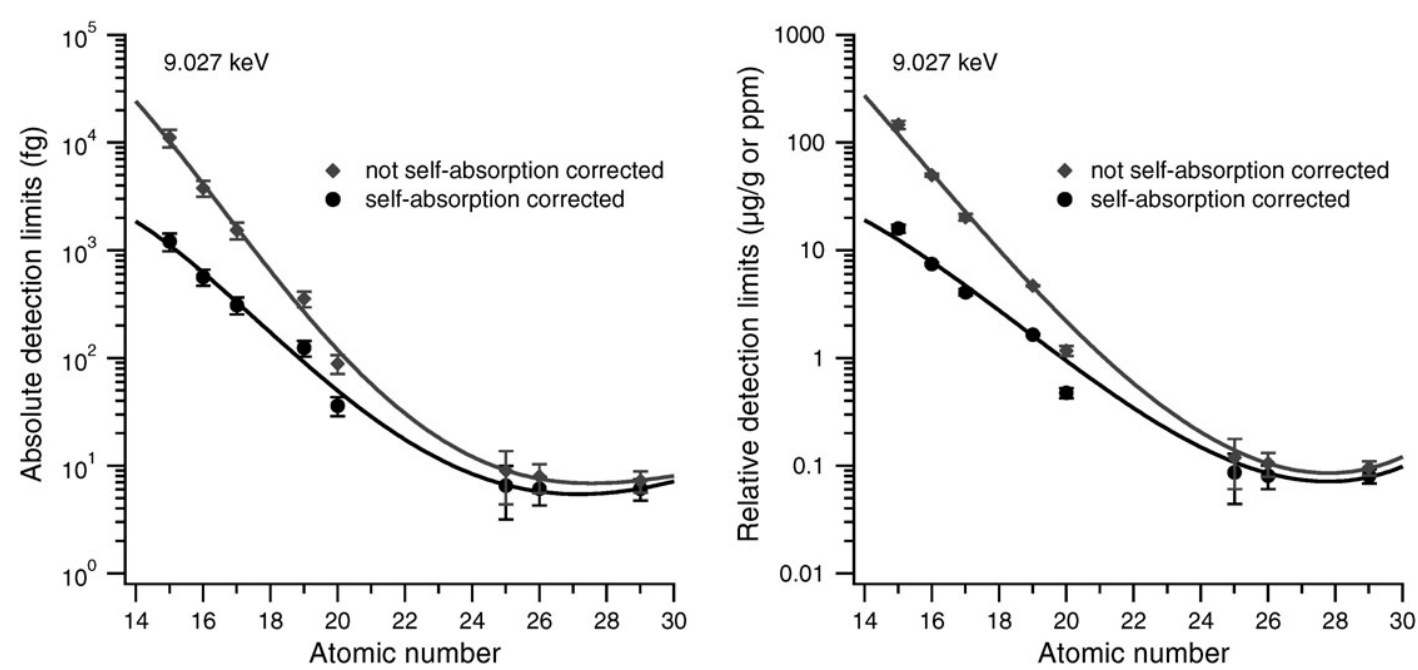

Fig. 4 Elemental detection limits determined using the NIST SRM1577b standard for an incoming X-ray energy of $9.027 \mathrm{keV}$ and a measuring time of $300 \mathrm{~s}$ for the conventional micro-fluorescence detection mode, left: corresponding absolute detection limits; right: corresponding relative detection limits (the error bars represent $\pm 1 \mathrm{SD}$ ).
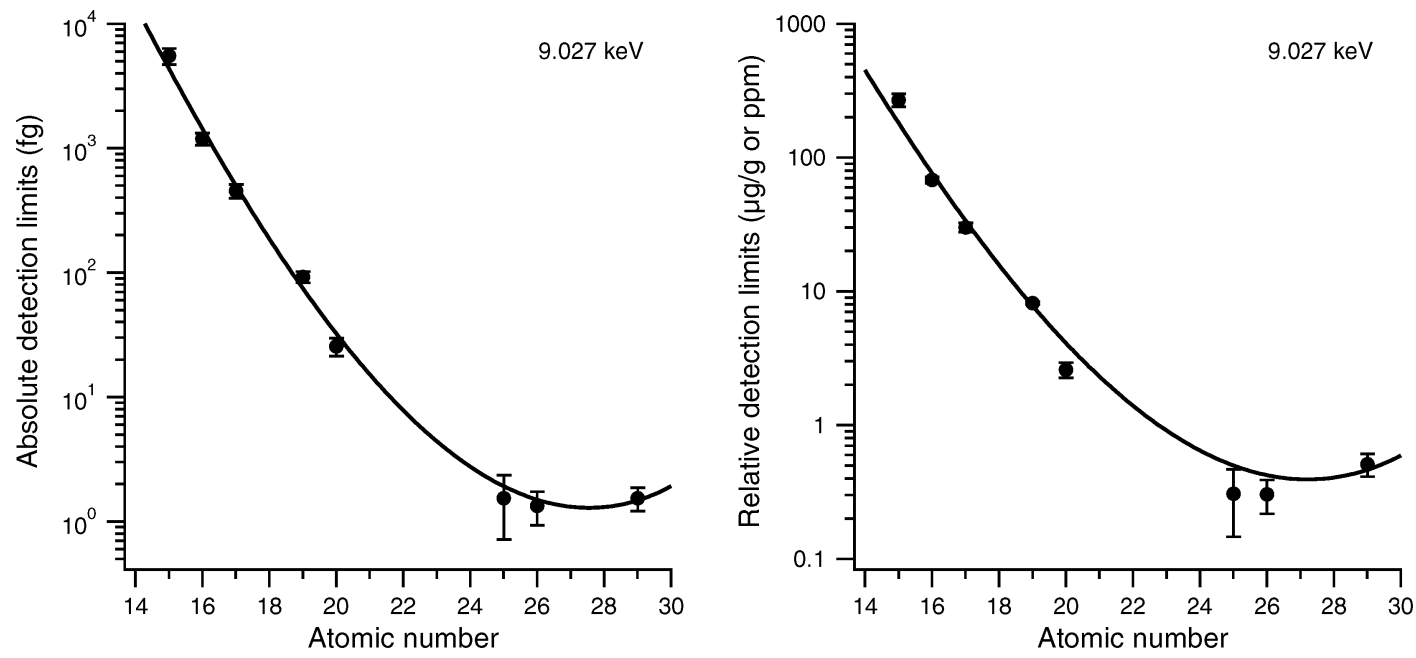

Fig. 5 Elemental detection limits determined using the NIST SRM1577b standard for an incoming X-ray energy of $9.027 \mathrm{keV}$ and a measuring time of $300 \mathrm{~s}$ for the confocal micro-fluorescence detection mode, left: corresponding absolute detection limits; right: corresponding relative detection limits (the error bars represent $\pm 1 \mathrm{SD}$ ).

(thickness $\sim 125 \mu \mathrm{m}$ ) is about $1.8 \times 10^{8} \mu \mathrm{m}^{3}$, giving an effective probed $\mathrm{Zn}$ mass of about $22 \mathrm{ng}$ (taking into account self-absorption effects). The intersection volume of the $\mu$-beam with the pellet is about $5.4 \times 10^{4} \mu^{3}$, with an effective detected $\mathrm{Zn}$ mass of about $6.7 \mathrm{pg}$. The confocal detection mode further reduces the detected volume to about $2.0 \times 10^{3} \mu \mathrm{m}^{3}$ with an effective detected $\mathrm{Zn}$ mass of about $290 \mathrm{fg}$.

\section{Applications: mineral inclusions in natural diamonds}

The majority of the natural diamonds are formed in the Earth's lithospheric upper mantle ( $<200 \mathrm{~km}$ depth). During the growth of the diamond, fluids, minerals and rock fragments can be trapped inside it. These inclusions are then shielded from the environment during the transport of the diamond towards the Earth surface preserving their original capture composition. Only a few sources, e.g. Juina (Brazil) and Kankan (Guinea), provide so called "ultra-deep" rare diamonds which are formed in the transition zone and the lower mantle regions of the deep Earth at depths of several hundreds of $\mathrm{km}$. These unique diamonds and their inclusions form the only direct source of information available on the physical and chemical conditions in the deep Earth down to at least the upper part of the lower mantle. ${ }^{7-17}$ In order to preserve the enclosed nature of the inclusions a non-destructive in situ analysis method is desired. In this respect X-ray techniques like XRF, XRD and XAS can be very useful.

However, due to the high X-ray scattering power of the diamond host a confocal detection mode is needed in order to examine the mostly complex, polyphase inclusions inside the diamond with XRF. Indeed, confocal $\mu$-XRF has been shown to allow the $3 \mathrm{D}$ visualisation of main and trace elements within $\mathrm{a} \sim 150 \mu \mathrm{m}$ large mineral inclusion. ${ }^{17}$ Conventional 


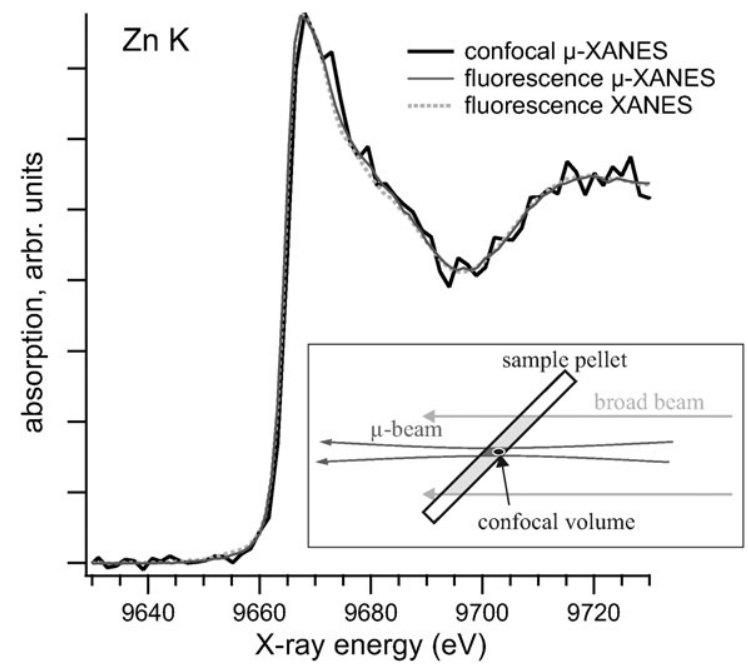

Fig. 6 Zn K-edge XAS spectra on NIST SRM1577b obtained in the conventional fluorescence mode (broad beam) and conventional and confocal $\mu$-XANES in fluorescence detection. The inset shows a top view sketch of the resulting different detection volumes for the three fluorescence detection modes.

and confocal $\mu$-XRF spectra recorded on the same mineral inclusion studied in ref. 17 are given in Fig. 7. While the confocal mode allows the detection of the XRF K-lines from $\mathrm{Ca}$ to $\mathrm{Zr}$, barely any lines are visible in the conventional detection mode due to the overwhelming scatter intensity of the X-ray beam originating from the diamond matrix, showing the tremendous importance of using the confocal detection mode for the in situ study of mineral inclusions enclosed in diamonds.

For the confocal analysis, a window is polished in the diamond very close to the respective inclusions (in the order of hundreds of $\mu \mathrm{m}$ or less). The diamond is then mounted with the polished surface mounted vertically and placed under an angle of $45^{\circ}$ with respect to the incoming $\mu$-beam and the detector axis. In order to locate the exact position of the inclusion within the diamond host, a horizontal or vertical confocal $\mu$-XRF map is performed first giving an in-depth elemental distribution maps perpendicular to the polished surface.

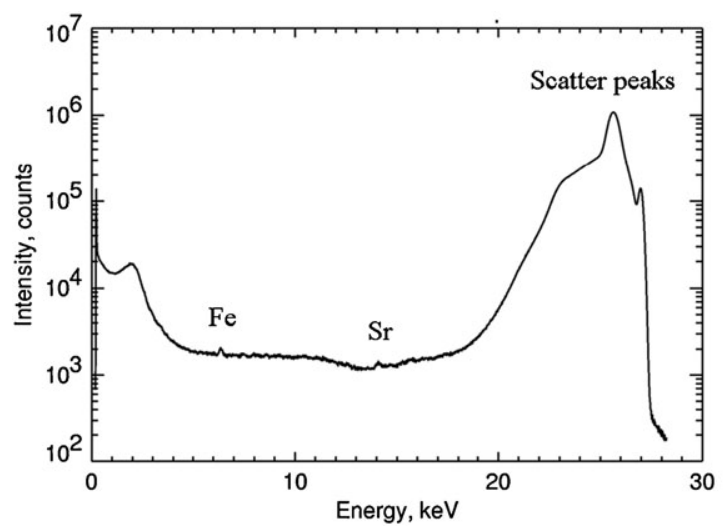

As an application of the confocal $\mu$-XAS method we present below depth-selective Fe K $\mu$-XANES on microscopic mineral inclusions trapped in "ultra-deep" diamonds from the Kankan (Guinea) and from Juina (Brazil) region.

\subsection{Kankan diamond}

As a first example a diamond from the Kankan region (Guinea) is studied. This "super-deep" diamond originates from the upper part of the lower mantle of the Earth down to depths exceeding $670 \mathrm{~km}$. The elemental distributions of $\mathrm{K}$, $\mathrm{Ca}$ and $\mathrm{Fe}$ for a horizontal plane scan inside the diamond (KK106c) containing mineral inclusions are given in Fig. 8 (dimensions for the ellipsoidal detection volume are $\left.20.5 \times 18.5 \times 14.0 \mu \mathrm{m}^{3}\left(D_{\mathrm{B}} \times D_{\mathrm{A}} \times D_{\mathrm{V}}\right)\right)$. Combining these maps allows the identification of 3 possible inclusions, indicated on the elemental distributions in Fig. 8. The corresponding confocal $\mathrm{Fe} \mathrm{K} \mu$-XANES spectra for these three locations are given in Fig. 9. The Fe K XANES spectrum taken on the very intense $\mathrm{Fe}$ spot (\#1) has a clear metallic fingerprint, and thus points towards a metallic inclusion. Region \#2 is a mineral inclusion and the measured $\mathrm{Fe} \mathrm{K}$ XANES spectrum agrees well with the reference spectrum of Spessartite, a $\mathrm{Fe}$ containing garnet. The $\mathrm{Fe} \mathrm{K}$ XANES spectrum taken on the third region (\#3) is a mixture of the two other recorded spectra, region \#3 also contains a mineral inclusion, but it is close enough to the metallic grain to pick up a fraction of its much higher intensity.

\subsection{Juina diamond}

A second "ultra-deep" diamond from the Juina region (Matto Grosso State, Brazil) has been studied at beamline L (HASYLAB). The measured dimensions (FWHM) for the ellipsoidal confocal detection volume at the $\mathrm{Fe} \mathrm{K}$ edge for this beamline were $21 \times$ $26 \times 17 \mu \mathrm{m}^{3}\left(D_{\mathrm{B}} \times D_{\mathrm{A}} \times D_{\mathrm{V}}\right)$. This diamond (RS69) contains a stream of $1-10 \mu \mathrm{m}$ sized mineral inclusions, as can be seen on the Fe elemental distribution map shown in Fig. 10. Confocal Fe K $\mu$-XANES spectra were recorded on a selection of these inclusions which revealed a change in the mineralogical compositional along the inclusion stream. Representative spectra for the upper part and the lower part of the map are given in Fig. 11. The spectrum for the lower part is identified as originating mainly from hematite

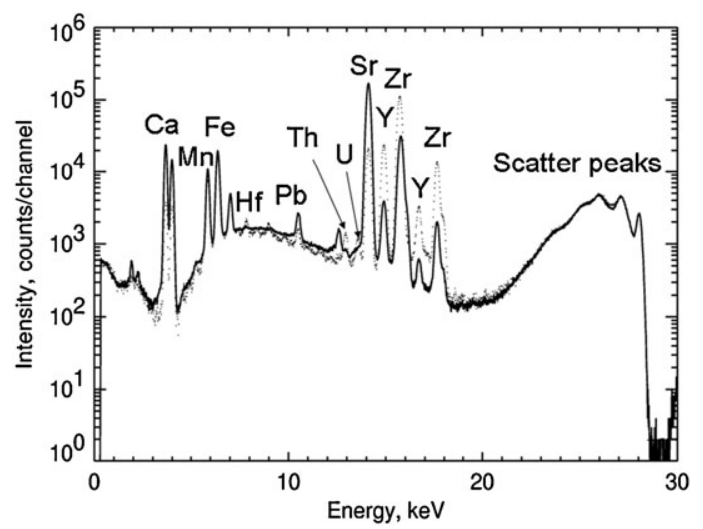

Fig. 7 Conventional (left) and confocal (right) $\mu$-XRF spectra from a $\sim 150 \mu \mathrm{m}$ mineral inclusion within a natural diamond host (Kankan district, Guinea) studied by confocal $\mu$-XRF in ref. 17 . 

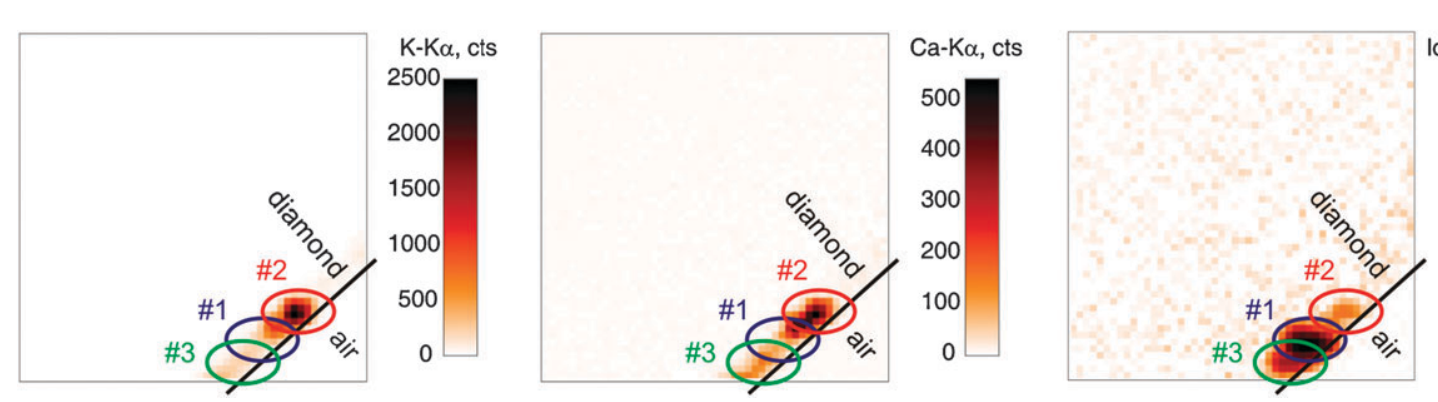

Fig. 8 2D elemental distributions of $\mathrm{K}, \mathrm{Ca}$ and $\mathrm{Fe}$ for a horizontal plane within a Kankan diamond containing mineral inclusions, obtained with confocal $\mu$-XRF $(51 \times 10 \mu \mathrm{m} \times 51 \times 10 \mu \mathrm{m})$.
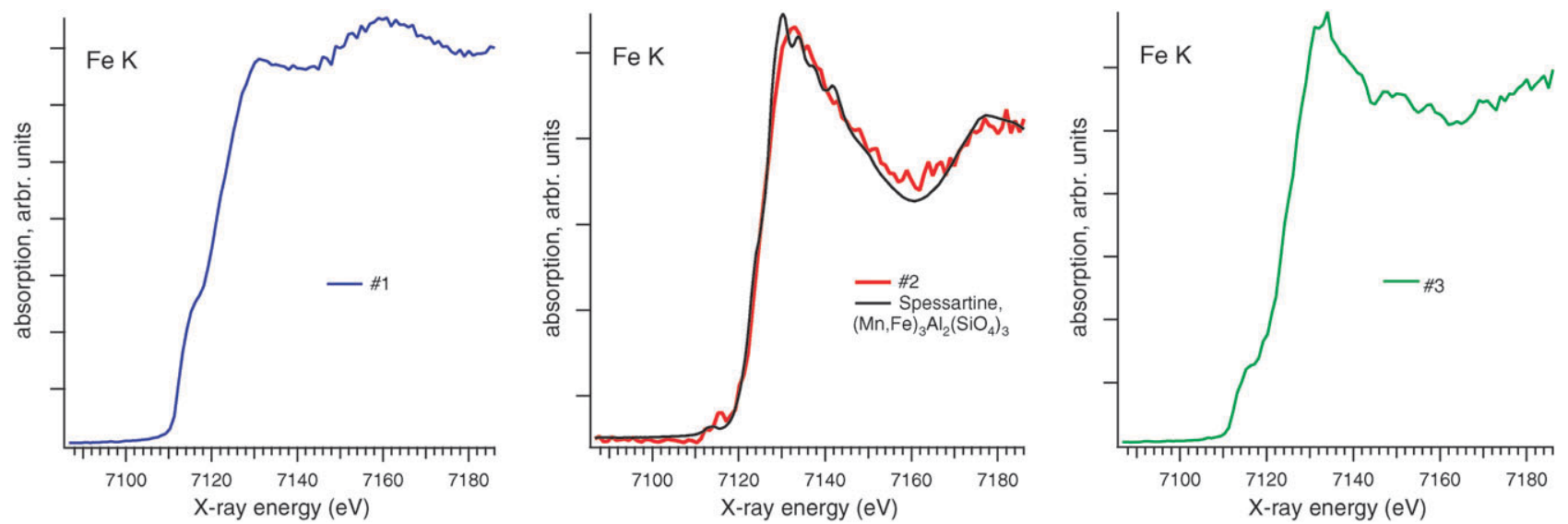

Fig. 9 Confocal Fe K $\mu$-XANES spectra of the regions indicated in Fig. 8.

$\left(\mathrm{Fe}_{2} \mathrm{O}_{3}\right)$. The spectrum for the upper part is not yet unambiguously identified, but from comparison with Fe XANES reference spectra given by Wilke et al., ${ }^{18}$ the measured spectrum closely resembles to that of a periclase mineral (the cubic form of $\mathrm{MgO}$ ). Moreover, from the set of Fe bearing minerals which we have at our disposal as reference (excluding periclase), the best agreement was found for forsterite $\left(\mathrm{Mg}_{2} \mathrm{SiO}_{4}\right)$, again a $\mathrm{Mg}-\mathrm{O}$ containing compound.

\section{Outlook}

Polycapillary based confocal fluorescence $\mu$-XAS allowing 3D resolved micro analysis has been characterized and illustrated with a depth-selective Fe K $\mu$-XANES study on microscopic

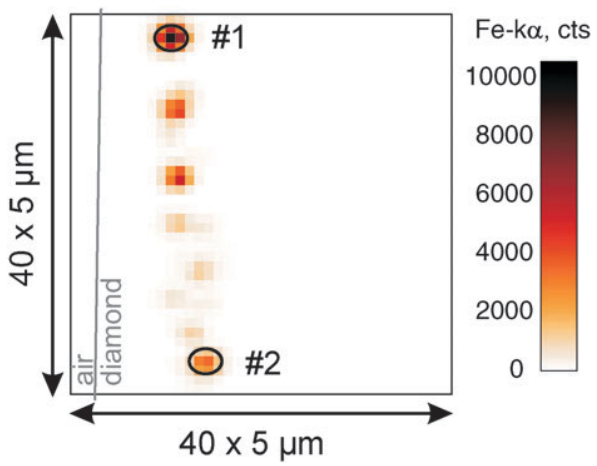

Fig. 10 2D elemental distribution of Fe for a vertical plane within a Juina diamond containing a stream of microscopic mineral inclusions, obtained with confocal $\mu$-XRF. inclusions trapped inside natural diamonds. These first results show that a systematic study of inclusions from a larger set of diamonds can provide valuable information on the physical and chemical conditions of the deep Earth.

More generally, like confocal $\mu-\mathrm{XRF}$, confocal $\mu$-XAS has a great potential in the study of high $Z$ elements (from the $3 \mathrm{~d}$ transition metals on) in weakly absorbing and/or strongly scattering low $Z$ matrices. Besides the diamond example given in the current work, confocal $\mu$-XAS can be a valuable technique for the local speciation of transition metals in different research fields such as biology, ecotoxicology, geology, earth and planetary sciences and palaeontology. Possible samples include inclusions in gemstones or quartz (geology) and insects in amber (palaeontology). Especially in biological and ecotoxicological systems confocal $\mu$-XAS can give useful complementary information. As a specific example, element-to-tissue correlation in Daphnia magna has been demonstrated with $\mu$-XRF and confocal $\mu-X R F,{ }^{5-6,19}$ confocal $\mu$-XAS can add (intra-)tissue-specific metal speciation knowledge to this elemental information.

\section{Conclusions}

Three dimensional spatially resolved fluorescence $\mu$-XAS in a confocal detection scheme based on polycapillary half-lenses has been demonstrated and characterized. The confocal detection volume is an ellipsoid with main axes FWHM of $20.5 \times 18.5 \times 14.0 \mu \mathrm{m}^{3}$ at the Fe K-edge. Relative detection limits for the $3 \mathrm{~d}$ transition metals of less than $1 \mathrm{ppm}$ and 

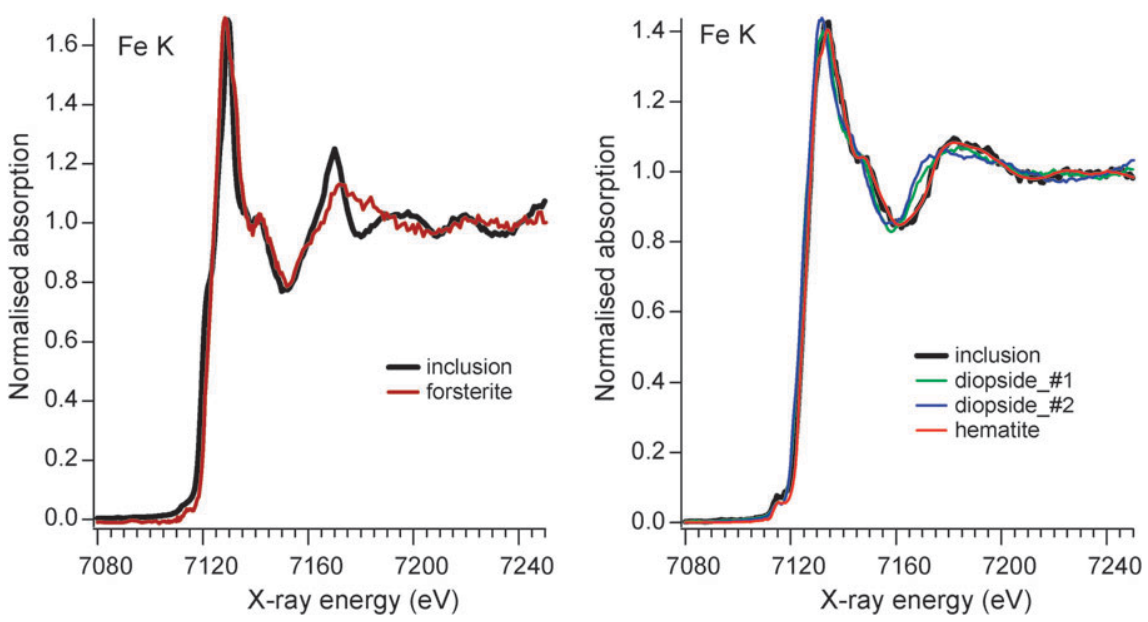

Fig. 11 Confocal Fe K $\mu$-XANES spectra of the inclusion \#1 (left) and \#2 (right) indicated on Fig. 10.

absolute detection limits of a few fg were obtained when measuring for $300 \mathrm{~s}$. The $\mathrm{Zn} \mathrm{K} \mu$-XANES spectra recorded on the NIST SRM1577b standard in the confocal detection agrees will with the conventional XANES (broad beam) or $\mu$-XANES spectra, showing that the confocal detection mode does not introduce distortions in the XANES spectrum. The confocal detection mode allowed to perform depth-selective $\mu$-XANES on microscopic inclusions trapped within a strongly scattering diamond host, and to identify the inclusion phases inside natural diamonds from the Kankan and Juina region.

\section{Acknowledgements}

Dr G. Silversmit is supported by a postdoctoral fellowship from the Research Foundation-Flanders (FWO-Vlaanderen, Belgium). This research was performed as part of the Interuniversity Attraction Poles (IAP6) programme financed by the Belgian Government. This work was further supported by HASYLAB at DESY within the initiative "ELISA: EU Support of Access to Synchrotrons/FELs in Europe". FWO-NWO are thanked for making the beamtime on the DUBBLE beamline available. We kindly thank the staff of DUBBLE and of HASYLAB-L for the technical support. We like to thank Thomas Stachel, Jeff Harris and Felix Kaminsky for providing the diamond samples and Prof. Dr M. Elburg for providing the reference minerals used in this study.

\section{References}

1 G. Silversmit, B. Vekemans, S. Nikitenko, W. Bras, V. Czhech, G. Zaray, I. Szaloki and L. Vincze, J. Synchrotron Radiat., 2009, 16, 237-246.

2 W. Malzer and B. Kanngiesser, Spectrochim. Acta, Part B, 2005, 60, 1334-1341.

3 S. Nikitenko, A. M. Beale, A. M. J. van der Eerden, S. D. M. Jacques, O. Leynaud, M. G. O'Brien, D. Detollenaere, R. Kaptein, B. M. Weckhuysen and W. Bras, J. Synchrotron Radiat., 2008, 15, 632-640.
4 A. Brunetti, M. Sanchez del Rio, B. Golosio, A. Simionovici and A. Somogyi, Spectrochim. Acta, Part B, 2004, 59, 1725-1731.

5 B. De Samber, G. Silversmit, K. De Schamphelaere, R. Evens, T. Schoonjans, B. Vekemans, C. Janssen, B. Masschaele, L. Van Hoorebeke, I. Szaloki, F. Vanhaecke, K. Rickers, G. Falkenberg and L. Vincze, J. Anal. At. Spectrom., 2010, 25, 544-553.

6 B. De Samber, R. Evens, K. De Schamphelaere, G. Silversmit, B. Masschaele, T. Schoonjans, B. Vekemans, C. R. Janssen, L. Van Hoorebeke, I. Szalóki, F. Vanhaecke, G. Falkenberg and L. Vincze, J. Anal. At. Spectrom., 2008, 23, 829-839.

7 T. Stachel, G. P. Brey and J. W. Harris, Contrib. Mineral. Petrol., $2000,140,1-15$.

8 T. Stachel, J. W. Harris, G. P. Brey and W. Joswig, Contrib. Mineral. Petrol., 2000, 140, 16-27.

9 B. Harte, J. W. Harris, M. T. Hutchison, G. R. Watt and M. C. Wilding, in Lower mantle mineral associations in diamonds from Sao Luiz, Brazil, ed. Y. Fei, C.M. Bertka and B.O. Mysen, Mantle Petrology: Field Observations and High Pressure Experimentation: A tribute to Francis R. (Joe) Boyd, Geochemical Society, USA, 1999, vol. 6, pp. 125-153.

10 T. Stachel, Eur. J. Mineral., 2001, 13, 883-892.

11 F. Kaminsky, O. D. Zakharchenko, R. Davies, W. L. Griffin, G. K. Khachattryan-Blinova and A. A. Shiryaev, Contrib. Mineral. Petrol., 2001, 140, 734-753.

12 P. Hayman, M. Kopylova and F. Kaminsky, Contrib. Mineral. Petrol., 2005, 149, 430-445.

13 R. Tappert, T. Stachel, J. W. Harris, K. Muehlenbachs, T. Ludwig and G. P. Brey, Geology, 2005, 33, 565-568.

14 F. E. Brenker, T. Stachel and J. W. Harris, Earth Planet. Sci. Lett., 2002, 198, 1-9.

15 R. Wirth, C. Vollmer, F. E. Brenker, S. Matsyuk and F. Kaminsky, Earth Planet. Sci. Lett., 2007, 259, 384-399.

16 F. E. Brenker, L. Vincze, B. Vekemans, L. Nasdala, T. Stachel, C. Vollmer, M. Kersten, A. Somogyi, F. Adams, W. Joswig and J. W. Harris, Earth Planet. Sci. Lett., 2005, 236, 579-587.

17 L. Vincze, B. Vekemans, F. E. Brenker, G. Falkenberg, K. Rickers, A. Somogyi, M. Kersten and F. Adams, Anal. Chem., 2004, 76(22), 6786-6791.

18 M. Wilke, F. Farges, P.-E. Petit, G. E. Brown Jr. and F. Martin, Am, Mineral,, 2001, 86, 714-730.

19 B. De Samber, G. Silversmit, R. Evens, K. De Schamphelaere, C. Janssen, B. Masschaele, Van Hoorebeke, L. Balcaen, F. Vanhaecke, G. Falkenberg and L. Vincze, Anal. Bioanal. Chem., 2008, 390, 267-271. 